\title{
Solid immersion lens at the aplanatic condition for enhancing the spectral bandwidth of a waveguide grating coupler
}

\author{
Marcelo B. Pereira \\ Instituto de Física \\ UFRGS \\ CP 15051
}

Porto Alegre, RS 91501-970, Brazil

\author{
Jill S. Craven \\ California Institute of Technology \\ Department of Chemical Engineering \\ Pasadena, California 91125
}

Sergio B. Mendes

University of Louisville

Department of Physics and Astronomy

Louisville, Kentucky 40292

E-mail: sbmend01@louisville.edu

\begin{abstract}
We report a technique to substantially boost the spectral bandwidth of a conventional waveguide grating coupler by using a solid immersion cylindrical lens at the aplanatic condition to create a highly anamorphic beam and reach a much larger numerical aperture, thus enhancing the spectral bandwidth of a free-space propagating optical beam coupled into a single-mode planar integrated optical waveguide (IOW). Our experimental results show that the broadband IOW spectrometer thus created almost doubles (94\% enhancement) the coupled spectral bandwidth of a conventional configuration. To exemplify the benefits made possible by the developed approach, we applied the technique to the broadband spectroscopic characterization of a protein submonolayer; our experimental data confirm the enhanced spectral bandwidth (around $380-\mathrm{nm}$ ) and illustrate the potentials of the developed technology. Besides the enhanced bandwidth, the broadband coupler of the single-mode IOW spectrometer described here is more robust and user-friendly than those previously reported in the literature and is expected to have an important impact on spectroscopic studies of surface-adsorbed molecular layers and surface phenomena. (C) 2010 Society of Photo-Optical Instrumentation Engineers. [DOI: 10.1117/1.3520063]
\end{abstract}

Subject terms: micro-optics; beam-shaping optics; grating coupler; biophotonics; optical spectroscopy; molecular films.

Paper 100554R received Jul. 8, 2010; revised manuscript received Oct. 13, 2010; accepted for publication Oct. 15, 2010; published online Dec. 7, 2010.

\section{Introduction}

Due to its outstanding effective path length for absorbance measurements in ultrathin films, integrated optical waveguides (IOWs) have become an increasingly important tool $^{1-17}$ for studying surface-confined molecular phenomena with potential applications in many technologies, such as electrochemical and biochemical sensors, affinity-based bioseparations, and materials biocompatibility. In particular, single-mode IOW platforms ${ }^{5,6,10-17}$ have been shown to produce far superior performance to that of competing approaches for detecting ultrathin films, such as attenuated total reflection, the quartz crystal microbalance, ellipsometry, and surface plasmon resonance. The use of a single-mode IOW platform combined with monochromatic light from a laser source for the investigation of a molecular layer adsorbed on the waveguide surface is straightforward and has been well established. ${ }^{5,6,12,17}$ However, the amount of information provided by monochromatic measurements is very limited and usually not suitable as a research tool aiming for more in-depth investigations of molecular films. For example, broadband spectroscopic information is greatly needed for spectral investigations to address conformational changes in protein films, redox couple states, angular orientation and order in surface-adsorbed biomolecules, etc. On the other hand, broadband coupling into a single-mode IOW presents challenges due to the dispersive nature of transverse couplers, which must match the effective refractive index of the

$\overline{0091-3286 / 2010 / \$ 25.00 \text { @ 2010 SPIE }}$ single-mode waveguide structure. Broadband coupling using a prism coupler, either with an increased angular width of the incoming beam ${ }^{10,13-16}$ or with an achromatic design, ${ }^{18}$ have been reported and employed for the spectroscopic interrogation of an adsorbed monolayer. However, prism coupling occurs through an evanescent field, which requires the prism to be placed in close proximity to the waveguiding film, separated by a very small (in the wavelength range) and precisely fixed gap. Changes in this gap during the course of the experiment affect the coupling efficiency substantially and perturb the spectroscopic measurements. In addition, each sample to be measured requires a precise prism and waveguide film alignment. A grating coupler approach combined with two predispersive components (one prism and one diffraction grating) to match the coupler-waveguide dispersion has been developed ${ }^{11,19}$ to deliver a spectral bandwidth greater than $100 \mathrm{~nm}$; the approach was successfully employed for the first demonstrations of an IOW spectrum of a protein submonolayer. ${ }^{11}$ Again, the critical alignment of the predispersive elements with the waveguide grating coupler is demanding and not trivial for applications beyond the research environment.

Grating couplers are devices fully embedded into the laminar structure of integrated optical waveguides, and that feature provides enormous robustness to the coupling process. However, due their strongly dispersion behavior, grating couplers require either an angular tuning mechanism or a large numerical aperture to reach broadband operation. Angular tuning is inconvenient, in that it slows data acquisition and brings concerns about repeatability. In this work, we describe 


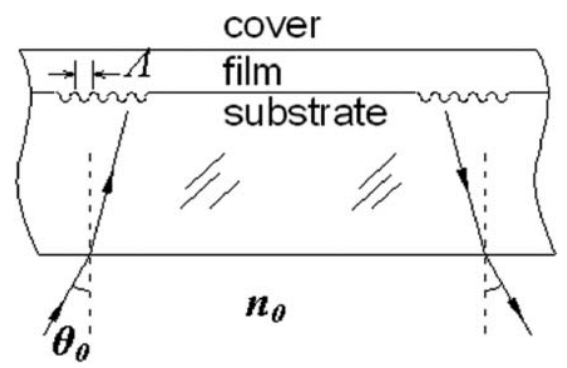

Fig. 1 Schematic structure of a planar integrated optical waveguide (IOW) with a pair of grating couplers.

a technique for enhancing the numerical aperture delivered to a grating coupler based on a solid immersion cylindrical lens working with a highly anamorphic optical beam at the aplanatic condition for broadband coupling. Our experimental results show a significant enhancement in the coupled spectral bandwidth. Moreover, the optical components shaping the light beam to be delivered to the grating coupler only require minor alignment, which makes the IOW spectrometer very reliable, reproducible, and user-friendly. To illustrate the potentials of the developed technology and to confirm its capability, we also report here experimental results on the spectroscopic characterization of a protein submonolayer.

\section{Experimental Setup}

In order to couple a light beam propagating in free space into an optical mode of a planar waveguide using a diffraction grating, the following well-known synchronous condition must be satisfied ${ }^{20}$ :

$N_{\text {eff }}=n_{0} \sin \theta_{0}+\frac{\lambda}{\Lambda}$,

where $N_{\text {eff }}$ is the effective refractive index of the waveguide, and $\lambda$ is the wavelength of the light in vacuum. As indicated in Fig. $1, n_{0}$ is the refractive index of air, $\theta_{0}$ is the angle between the incident light beam and the normal to the waveguide surface, and $\Lambda$ is the period of the grating coupler. The central wavelength $\lambda_{c}$ of the grating coupler, defined at $\theta_{0}=0 \mathrm{deg}$, is then given by

$\lambda_{c}=\Lambda N_{\mathrm{eff}}$,

and the spectral bandwidth provided by the grating coupler can be approximated by

$\Delta \lambda \approx 2 \Lambda \mathrm{NA}$,

where NA is the numerical aperture of the incident optical beam. Equation (2) indicates that the period of the grating coupler must be chosen according to the desired central wavelength to be coupled into a particular waveguide. And after the period of the grating coupler has been fixed, Eq. (3) shows that the spectral bandwidth is solely determined by the numerical aperture of the light beam striking the grating coupler. Moreover, the coupled bandwidth scales directly with the numerical aperture of the optical beam. Therefore, for spectroscopic applications where a large spectral range is always desirable, it is imperative to enhance the working NA delivered to a grating coupler in an IOW platform. For this purpose, we have developed a solid immersion lens to

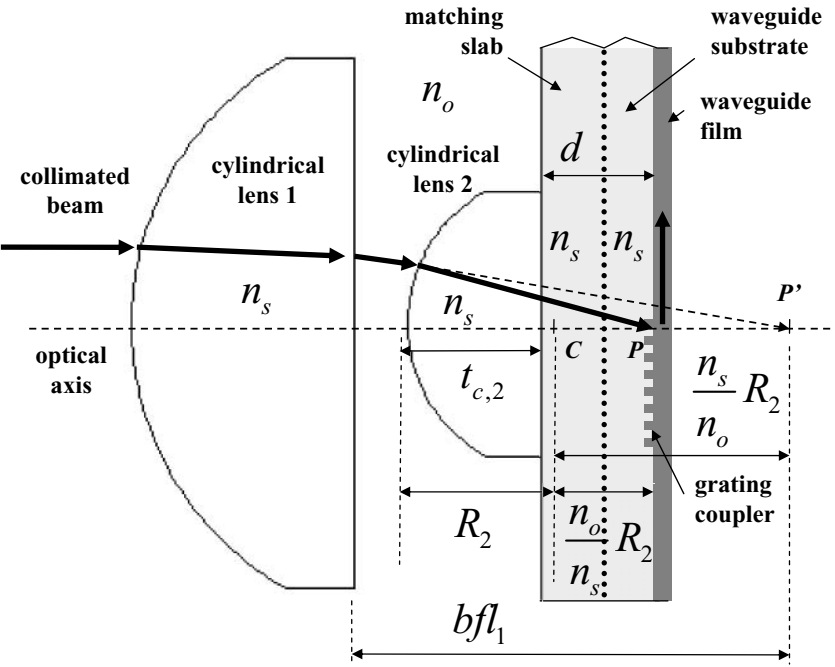

Fig. 2 Cross section of the aplanatic condition for the solid immersion lens of the grating coupler. The cylindrical surface of lens 2 (with center of curvature at $C$, radius $R_{2}$, center thickness $t_{c, 2}$, and refractive index $n_{s}$ ) has two aplanatic points: $P$ and $P^{\prime}$. The point $P$ is designed to be located at the diffraction grating coupler, and the point $P^{\prime}$ is designed to be located at the back focal point of the cylindrical lens 1 . The medium in between the cylindrical lens is air with refractive index $n_{0}$.

augment the numerical aperture of an incident beam to be coupled into a planar waveguide. By considering the slab waveguide geometry, we have opted for a highly anamorphic optical beam with large divergence (high NA) in the plane of incidence, but fairly collimated in the lateral direction of the planar optical waveguide. Furthermore, in order to keep the amount of spherical aberration low, the immersion lens unit was designed to operate at the aplanatic condition. Those considerations led us to the optical system sketched in Fig. 2. As indicated in the far left of Fig. 2, a collimated optical beam is bent towards the optical axis by the cylindrical lens 1 and propagates as a converging cylindrical wave. This cylindrical wave then impinges on the cylindrical lens 2, which is glued to the waveguide substrate to act as the solid immersion lens. In this particular situation, the cylindrical surface of lens 2 has two axial conjugate points, $P$ and $P^{\prime}$, with minimal spherical aberrations ${ }^{21}$; those points are called aplanatic points. The first aplanatic point, $P$, is chosen by design to be located at the diffraction grating coupler, while the second aplanatic point, $P^{\prime}$, is designed to be located at the back focal point of the cylindrical lens 1 . If we write $R_{2}$ for the radius of curvature of the cylindrical surface 2 , then we find that, as derived elsewhere, ${ }^{21}$ the distance from the center of curvature $C$ of the cylindrical surface of lens 2 to the aplanatic point $P$ is given by $\overline{C P}=\left(n_{0} / n_{s}\right) R_{2}$. On the other hand, the distance from $C$ to the other aplanatic point, $P^{\prime}$, is given by $\overline{C P^{\prime}}=\left(n_{s} / n_{0}\right) R_{2}$. Both of those expressions are based on the simplifying assumption that the two cylindrical lenses and the waveguide substrate are made from the same glass (i.e., have the same refractive index $n_{s}$ ) and that the medium between the two cylindrical lenses is air of refractive index $n_{0}$. Given those requirements, we searched for a convenient pair of cylindrical lenses that could match the aplanatic condition and deliver a high numerical aperture to the grating coupler. The lenses employed in this work are two cylindrical plano-convex lenses made of BK-7 glass with the 
Table 1

\begin{tabular}{|c|c|c|c|c|c|c|}
\hline Lens & $\begin{array}{l}\text { Melles Griot } \\
\text { cat. no. }\end{array}$ & efl (mm) & $\mathrm{bfl}(\mathrm{mm})$ & $\begin{array}{c}\text { Radius } \\
(\mathrm{mm})\end{array}$ & $\begin{array}{l}\text { Center } \\
\text { thickness } \\
(\mathrm{mm})\end{array}$ & NA \\
\hline 1 & 01LCP025 & 10.0 & 7.4 & 5.2 & 3.8 & 0.45 \\
\hline 2 & 01LCP023 & 5.0 & 3.5 & 2.6 & 2.3 & 0.45 \\
\hline
\end{tabular}

specification listed in Table 1. For this pair of lenses, we have calculated $\overline{C P}=1.7 \mathrm{~mm}, \overline{C P^{\prime}}=3.9 \mathrm{~mm}, d=2.0 \mathrm{~mm}$, and the separation between the two cylindrical lenses should be $0.9 \mathrm{~mm}$. The numerical aperture of the optical beam at the grating coupler then becomes NA $=1.04$, which represents a substantial enhancement over the individual elements. The thickness $d=2.0 \mathrm{~mm}$ to satisfy the aplanatic condition is actually formed by two BK-7 slabs of $1 \mathrm{~mm}$ each: one slab is the waveguide substrate containing a pair of surface relief gratings overcoated with the waveguide film, and the other one is a matching slab. The two slabs are connected by an optical gel $\left(n_{d}=1.520\right)$ from Cargille. The cylindrical lens 2 is attached to the matching slab using wax $\left(n_{d}=1.539\right.$, also from Cargille) to create an approximately homogeneous medium that extends from the cylindrical surface of lens 2 all the way to the diffraction grating coupler.

The IOW spectrometer setup that incorporates the grating coupler with the solid immersion lens approach is schematically shown in Fig. 3. An incoherent broadband light source (tungsten-halogen lamp, voltage $=6 \mathrm{~V}$, operating current $=$ $3.3 \mathrm{~A}$ ) is firstly focused with a pair of doublets (efl $=30 \mathrm{~mm}$, diameter $=25.4 \mathrm{~mm}$ ) onto a step-index, multimode optical fiber (core diameter $=600 \mu \mathrm{m}, \mathrm{NA}=0.38$ ). The light output at the distal end of the optical fiber is collimated by an aspherical lens (Thorlabs, efl $=11 \mathrm{~mm}, \mathrm{NA}=0.25$ ). The collimated beam is then delivered to the optics previously described for coupling the free-space propagating light beam into a single-mode planar IOW. An identical optical arrangement is used at the outcoupling port of the IOW device. For polarized data acquisition, a sheet polarizer is inserted in the optical path of the collimated beam as shown in Fig. 3. For comparison with the solid-immersion-lens approach, we also collected data for the case when no immersion lens was employed. Thus, in this configuration, the cylindrical lens 2 is removed from the optical path in both the input and output grating coupler ports. We note that the collimating aspherical lens of this particular experimental demonstration underfills the cylindrical lens 1 , so that the overall working NA, is 0.27 for the conventional configuration, and 0.64 for the unit with the solid immersion lens.

Two different spectroscopic setups were used in the measurements. In scheme A shown in Fig. 3, the output light is connected directly into an optical spectrum analyzer (OSA from Ando, AQ-6315A). In scheme B, the output light is sent to a monochromator (Spectra-Pro 2356, Acton-Princeton Instruments), where light intensity versus wavelength is measured by a CCD camera (Pixis 400B, Princeton Instruments). Both detection schemes are connected to a computer for data acquisition and analysis.

The single-mode IOW film was made of $\mathrm{Al}_{2} \mathrm{O}_{3}$, using an atomic layer deposition (ALD) process, by a Savannah-100 tool from Cambridge NanoTech Inc. Trimethyl aluminum (TMA, from Sigma Aldrich) and deionized water were employed as precursors to form the waveguide film; details of the process and parameters used can be found elsewhere. ${ }^{22}$ Measurements made with a Metricon 2010/M prism coupler, using a He-Ne laser and several argon-ion laser lines, allowed us to obtain the waveguide's physical thickness $(233 \mathrm{~nm})$ and calculate its effective refractive index for the wavelength range between 400 and $700 \mathrm{~nm}$.

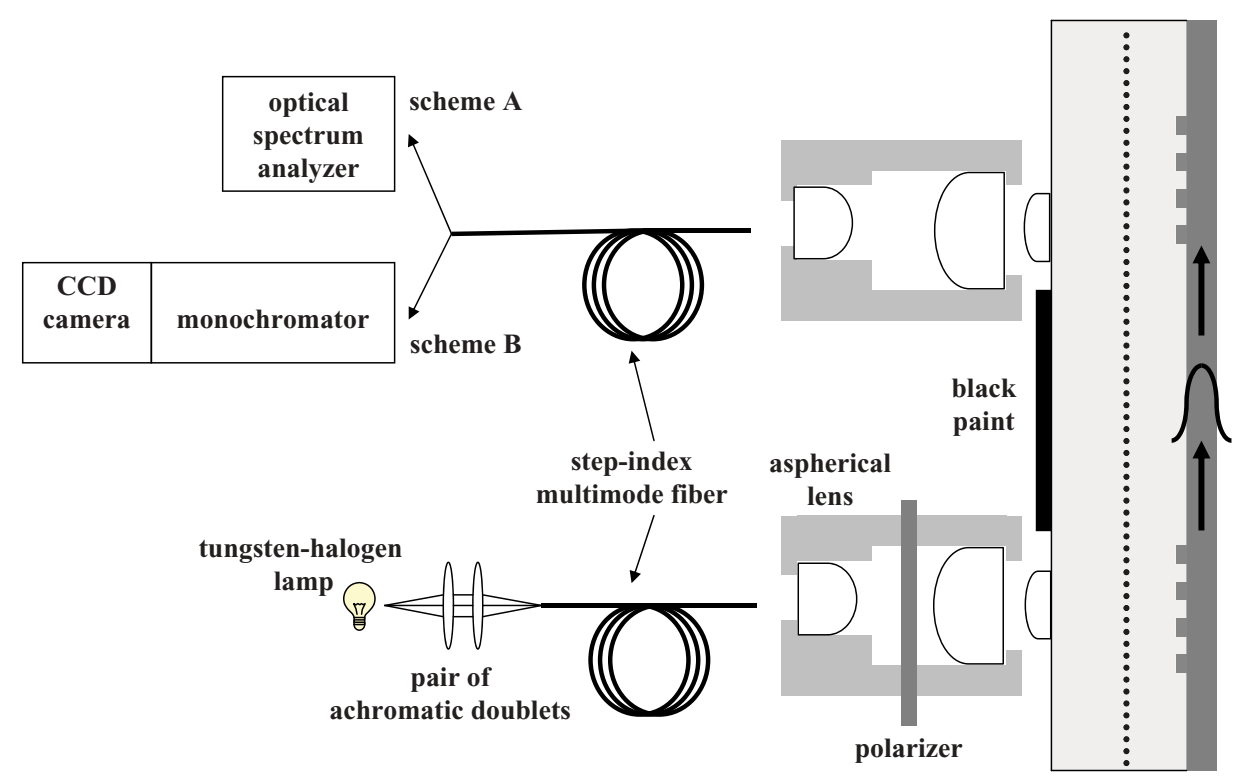

Fig. 3 Schematic view of the single-mode IOW spectrometer. The emitted light from a tungsten-halogen lamp is focused onto a multimode fiber by a pair of achromatic doublet lenses. The output light from the multimode fiber is then collimated by an aspherical lens. Then, a pair of cylindrical lenses, as described in Fig. 2, is used to focus the optical beam into a line parallel to the diffraction grating grooves. The outcoupling and incoupling optics are identical with the exception of a polarizer. Two sets of spectroscopic detectors were used: In scheme A the light output from the fiber was connected directly into an optical spectrum analyzer (OSA); in scheme B the light output was focused (optical components not shown) to the entrance slit of a monochromator connected to a CCD camera. 
As a demonstration of the broadband capability enabled by the developed approach, we performed spectroscopic measurements of a protein film adsorbed onto the surface of the waveguide film. In order to perform these measurements with the protein film maintained at an aqueous solution under neutral $\mathrm{pH}$, a 2-ml flow-cell device (not shown) was mounted on top of the waveguide film. The flow cell then allowed us to change solutions on the waveguide surface without disturbing the optical alignment.

\section{Experimental Results}

\subsection{Characterization of IOW Coupler Bandwidth}

Measurements of the spectral bandwidth of the IOW coupler were performed using scheme A shown in Fig. 3. Surface relief diffraction gratings with a spatial period (pitch) of $400 \mathrm{~nm}$ were used for the data presented here, and details of the fabrication process can be found elsewhere. ${ }^{22}$ To avoid stray light that would degrade the genuine signal, the rear surface of the substrate was coated with a black strippable paint in the region between the grating couplers, as indicated in Fig. 3.

The spectral density as collected for both the conventional configuration and the solid immersion lens is summarized in Fig. 4. In both cases, the coupled bandwidth was considered at the level of $10 \mathrm{~dB}$ below the maximum intensity in the spectral density. In the conventional configuration, the measurement was taken at approximately $-94 \mathrm{dBm} / \mathrm{nm}$ and the spectral bandwidth coupled to the single-mode IOW was $136.2 \mathrm{~nm}$, corresponding to the wavelength range between 542.8 and $679 \mathrm{~nm}$. On the other hand, for the solidimmersion-lens configuration, the bandwidth was taken at $-96 \mathrm{dBm} / \mathrm{nm}$ and gave a coupled spectral range of $264 \mathrm{~nm}$, corresponding to the region from 477.6 to $741.6 \mathrm{~nm}$. Thus, the improvement obtained using the solid immersion lens corresponds to an increase of about $94 \%$ in the optical bandwidth of the IOW coupler compared to the conventional configuration. This enhancement in bandwidth is highly relevant for several spectroscopic studies of surface-adsorbed molecules and interfacial phenomena.

Next, we performed a stray-light test to evaluate if the signal collected with the solid-immersion-lens grating couplers truly originated from the guided single-mode wave traveling along the waveguide. In this measurement, after an initial throughput signal had been acquired, a line of black ink was traced over the waveguide surface in the optical path between the grating couplers, and a stray light signal was acquired. The ink line absorbs almost entirely the light propagating as a surface guided mode, so any significant signal at this configuration would come from a spurious source other than the legitimate waveguide mode. As can be seen in Fig. 5, the signal collected in the stray-light measurement is extremely low in comparison with the output signal collected from the original guided mode. In fact, the stray-light signal is comparable to the residual noise from the detection equipment.

It is worthy noting that in the experimental setup described, an important aspect was the initial alignment of the optics to properly shape the optical beam toward the waveguide grating coupler, since this process determines the amount of broadband light coupled into the waveguide and the overall throughput of the instrument. For this purpose, an aluminum holder for each port (incoupling and outcoupling) was fabricated to mechanically align and fix together the optical fiber, the collimating lens, and the cylindrical lens 1 . The cylindrical lens 2 was aligned and attached to the matching slab, and this unit was combined with the glass slab containing the grating couplers and the waveguide film through an optical gel. An aluminum fixture accommodates those devices and keeps the slabs aligned with respect to each other. With those provisions, only the alignment between the aluminum blocks was required to reach efficient coupling, and this was accomplished very easily with one translation stage dedicated to each port (entrance and exit). In order to measure a new waveguide slab, one only requires unloading

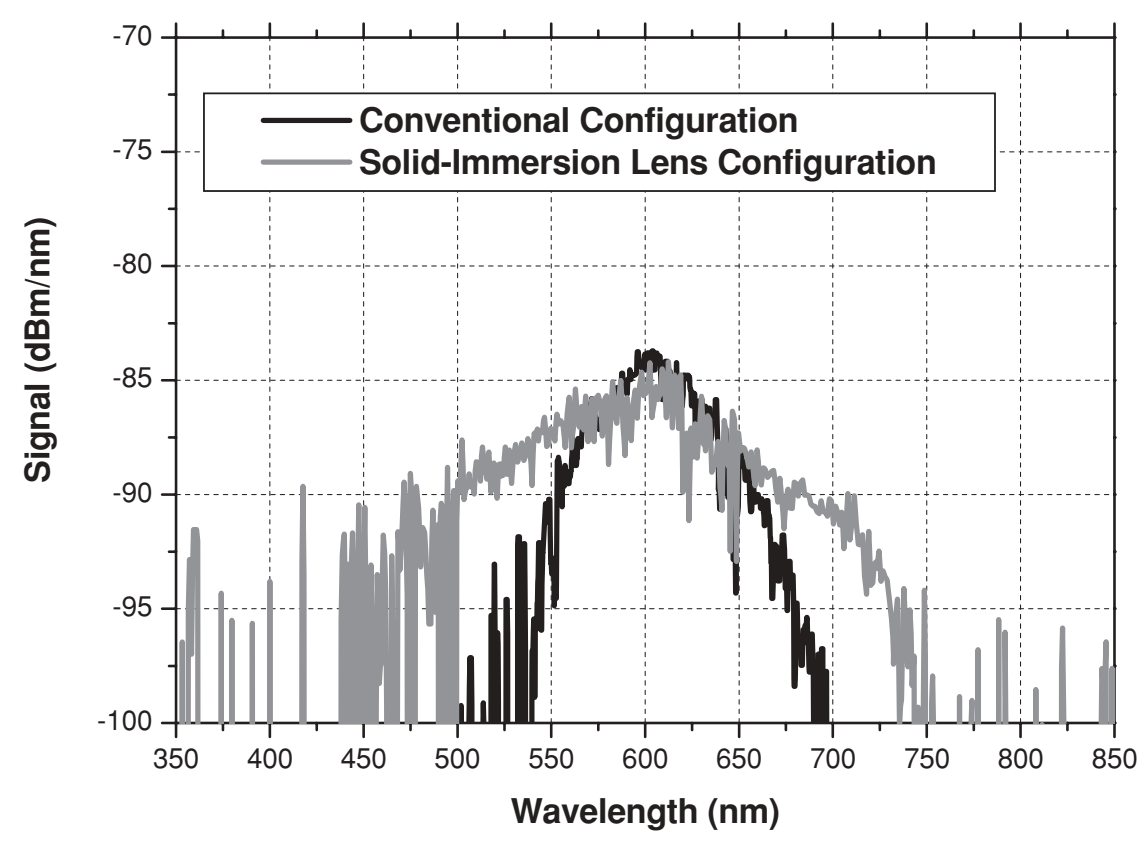

Fig. 4 The plot displays the output power density on a logarithmic scale: The black line is for the signal measured in the normal configuration, and the gray line is for the solid-immersion-lens configuration. 


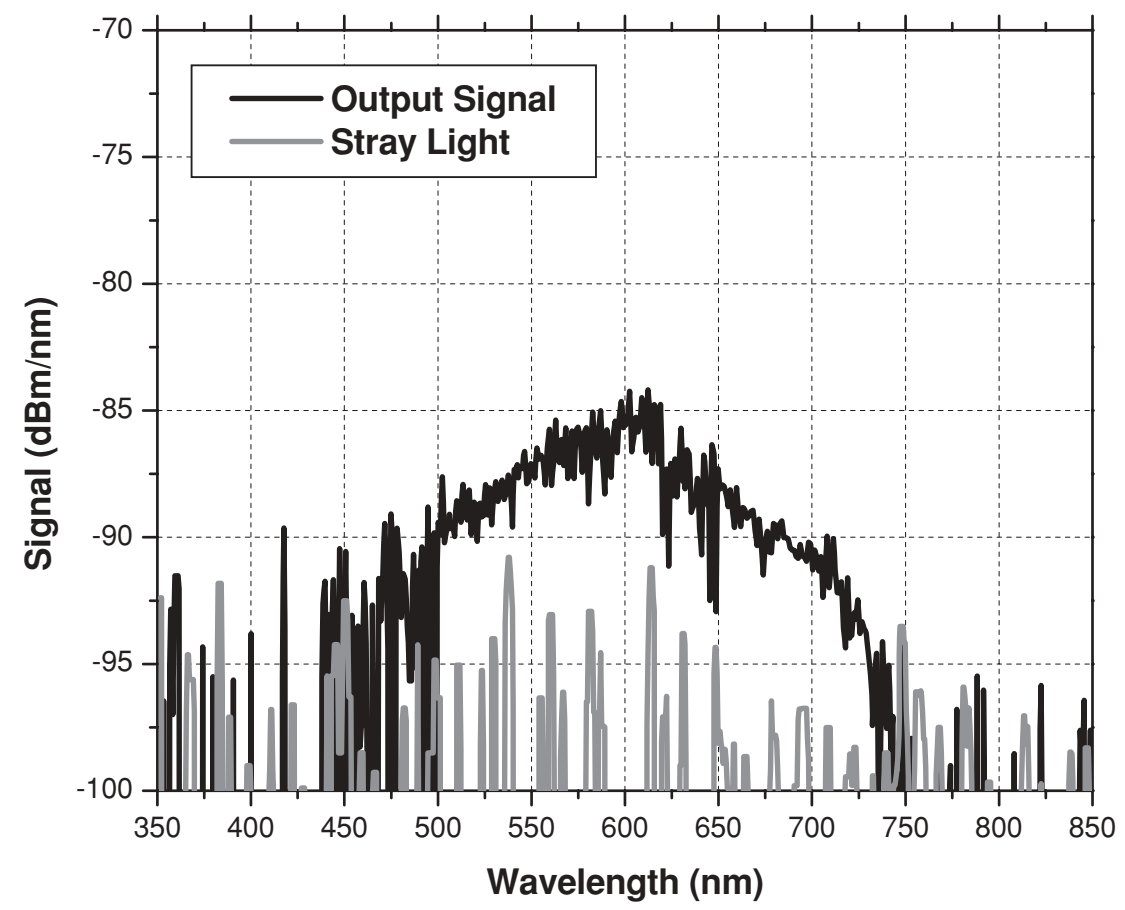

Fig. 5 Output signal (dark line) in comparison with stray-light signal (gray line). The stray-light measurement was performed after the waveguide surface was covered with a trace of black ink in the light path between the two gratings to eliminate the true guided mode.

the old sample and inserting the new one into its place at the aluminum fixture, with a minor tuning on the translation stages to reach good coupling. Unlike prism coupling and previously reported setups for broadband IOW experiments, the approach described here is highly reproducible and very user-friendly.

\subsection{Broadband IOW Spectra with the Solid-Immersion-Lens Grating Coupler}

To test the broadband capability of the solid-immersionlens grating coupler, absorbance measurements of a surfaceadsorbed protein submonolayer were performed using the single-mode IOW spectrometer. The measurements were made with detection scheme B shown in the setup of Fig. 3. The interaction path length $L$ used in these experiments was $31 \mathrm{~mm}$. A solution of bovine serum albumin (BSA), which was optically tagged with the Alexa $647 \mathrm{flu}-$ orophore (from Sigma Aldrich), was employed at a concentration of $5 \mathrm{ng} / \mathrm{ml}$ dissolved in sodium phosphate buffer at $\mathrm{pH}$ 7. For each experiment, a solution of pure buffer (no protein) was first injected into the flow cell to record a blank signal; then the protein solution was injected into the flow cell. Furthermore, a dark current measurement was taken at the end of experimental section (by applying a black ink trace on the waveguide surface) and subtracted from all signals during the data analysis.

In the first set of experiments, the BSA solution was injected into the waveguide flow cell and allowed to incubate for $5 \mathrm{~min}$, and then the cell was rinsed with buffer solution to remove any protein molecule that was not bound to the surface; after these steps data were acquired with the CCD camera. The absorbance spectrum of the surface-adsorbed protein layer obtained by the IOW spectrometer is shown in Fig. 6. As can be seen, by using the solid-immersion-lens approach, the spectral bandwidth obtained by the single-mode IOW spectrometer was quite large $(378 \mathrm{~nm})$ and allowed us to fully resolve the absorbance spectrum of the optically tagged BSA layer. In addition to the large bandwidth, it is important to note that the surface concentration of this protein layer is extremely low (lower than $100 \mathrm{pg} / \mathrm{cm}^{2}$, as described below) and the fact that the single-mode IOW spectrometer allowed us to reach such a strong absorbance signal (peak absorbance of about 2.1) at this level of surface concentration confirms the superb enhancement provided by the guided wave at the single-mode configuration. For comparison purposes, an absorbance spectrum was collected by a standard UV-visible spectrophotometer (Varian CARY 300) of the same optically tagged BSA dissolved in the buffer solution, and the normalized spectrum is also presented in Fig. 6. Besides an experimental artifact around $610 \mathrm{~nm}$, the shapes of the two curves agree well, indicating the reliability of the measurements made by the single-mode IOW spectrometer.

In the second set of experiments, TE polarized light was used to quantify the time evolution of the protein surface coverage, $\Gamma$, adsorbed to the $\mathrm{Al}_{2} \mathrm{O}_{3}$ waveguide surface. As before, a $5-\mathrm{ng} / \mathrm{ml}$ solution of the previously described protein was injected into the flow cell, and a sequence of timeresolved data was acquired by the CCD camera. The first data acquisition was taken $12 \mathrm{~s}$ after the protein solution was injected into the flow cell, and the last one $2 \mathrm{~h}$ after the protein injection. The waveguide absorbance, $A_{\mathrm{wg}}$, due to molecules immobilized on the waveguide surface, measured by a propagating guided wave, can then be used to determine the molecular surface concentration $\Gamma$. For the TE polarization, they are related by ${ }^{23}$

$\Gamma=\frac{t_{\mathrm{eff}} N_{\mathrm{eff}}\left(n_{w}^{2}-n_{c}^{2}\right)}{\varepsilon 2 n_{l}\left(n_{w}^{2}-N_{\mathrm{eff}}^{2}\right) L} A_{\mathrm{wg}}$,

where $\varepsilon$ is the molar absorptivity of the adsorbed molecule; $n_{c}, n_{l}$, and $n_{w}$ are the refractive indices of the cover (aqueous solution), molecular layer (protein), and waveguide 


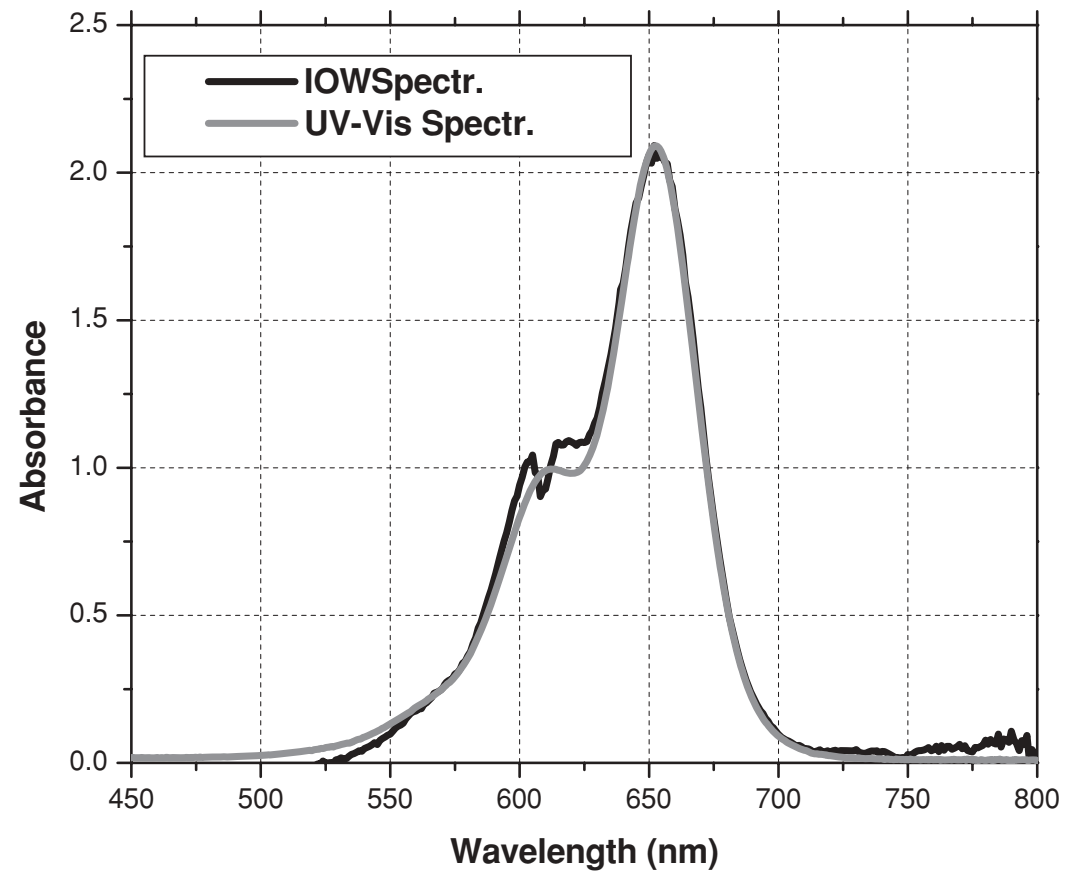

Fig. 6 Optical absorbance measurement of the optically tagged BSA protein using the single-mode IOW spectrometer (dark line) compared with the absorbance spectrum obtained by a standard UV-visible spectrophotometer (gray line).

film $\left(\mathrm{Al}_{2} \mathrm{O}_{3}\right)$, respectively; and $t_{\text {eff }}$ and $N_{\text {eff }}$ are the effective thickness and effective refractive index of the single-mode waveguide. The effective thickness $t_{\text {eff }}$ for a TE guided mode can be obtained from ${ }^{23}$

$t_{\text {eff }}=t+\frac{\lambda / 2 \pi}{\left(N_{\mathrm{eff}}^{2}-n_{c}^{2}\right)^{1 / 2}}+\frac{\lambda / 2 \pi}{\left(N_{\mathrm{eff}}^{2}-n_{s}^{2}\right)^{1 / 2}}$,

where $t$ is the physical thickness of the waveguide film, and $n_{s}$ is the refractive index of the waveguide substrate. The effective refractive index $N_{\text {eff }}$ at the fundamental waveguide mode can be found at each wavelength by numerically solving the following dispersion equation ${ }^{23}$ :

$$
\begin{aligned}
\frac{2 \pi}{\lambda} t\left(n_{w}^{2}-N_{\mathrm{eff}}^{2}\right)^{1 / 2}= & \tan ^{-1}\left(\frac{N_{\mathrm{eff}}^{2}-n_{c}^{2}}{n_{w}^{2}-N_{\mathrm{eff}}^{2}}\right)^{1 / 2} \\
& +\tan ^{-1}\left(\frac{N_{\mathrm{eff}}^{2}-n_{s}^{2}}{n_{w}^{2}-N_{\mathrm{eff}}^{2}}\right)^{1 / 2} .
\end{aligned}
$$

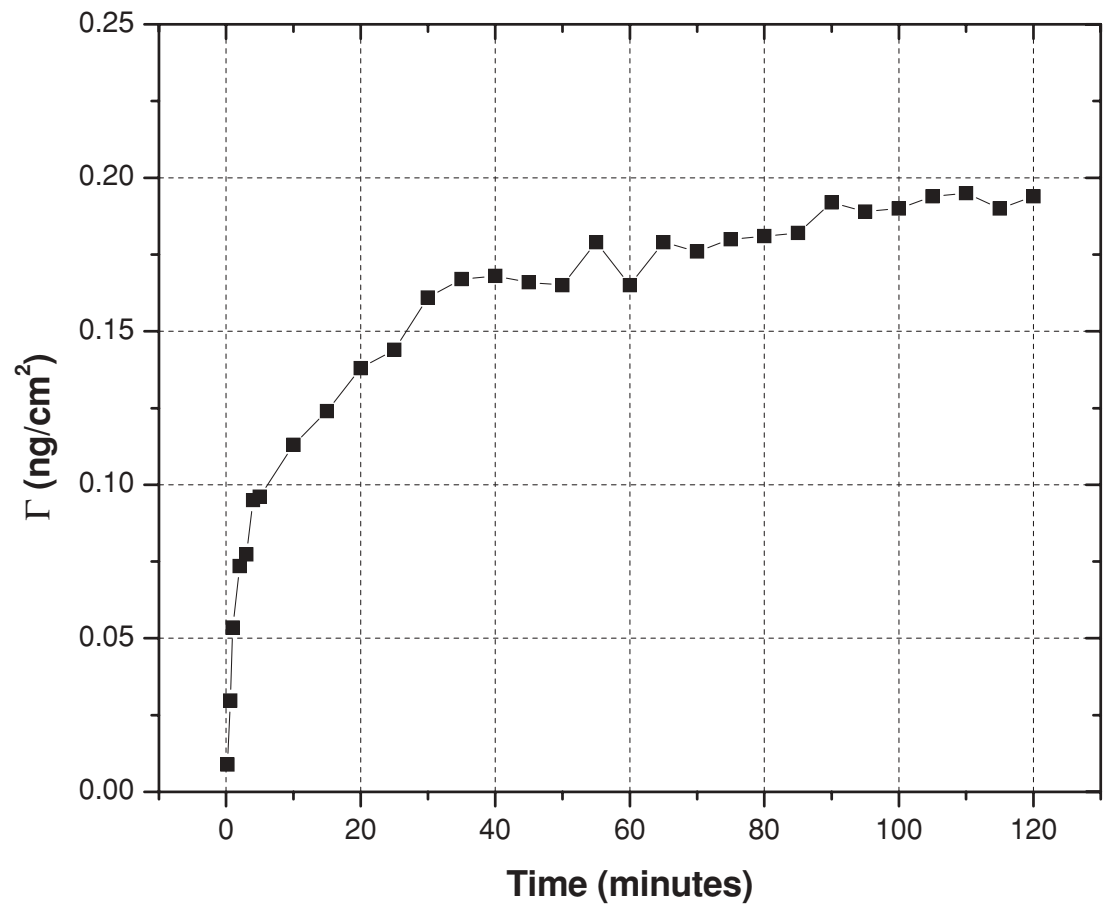

Fig. 7 Time evolution of the surface coverage $\Gamma$ of the protein BSA being adsorbed on the $\mathrm{Al}_{2} \mathrm{O}_{3}$ waveguide surface. 
The surface coverage was calculated using the $N_{\text {eff }}$ and $t_{\text {eff }}$ values corresponding to the wavelength of the absorbance peak from the optically tagged BSA, which has a maximum intensity located at the wavelength $650.8 \mathrm{~nm}$. The experimentally determined time evolution of the surface coverage, also known by the surface kinetics, is plotted in Fig. 7. The surface coverage follows a typical Langmuir profile with a steep initial increase and a gradual decrease in the slope after $5 \mathrm{~min}$ to reach steady state at approximately 90 min. The Langmuir profile can then be used to investigate surface binding interactions of the bound proteins, which however is beyond the scope of this report. Due to the high sensitivity of the IOW spectrometer, we note that it was possible to observe the time evolution of the molecular adsorption process since the first few seconds when $\Gamma \approx 10 \mathrm{pg} / \mathrm{cm}^{2}$. Again, those results reflect the superb sensitivity of the single-mode IOW spectrometer, and they are a few orders of magnitude better than the usual approaches employed in this field, such as the quartz crystal microbalance, ellipsometry, attenuated total reflection, and surface plasmon resonance.

\section{Conclusions}

We have described here a new approach for broadband coupling of a large spectral bandwidth into a single-mode IOW spectrometer; the approach is based on a highly anamorphic beam from a solid immersion lens working at the aplanatic condition to deliver a large numerical aperture to a diffraction grating coupler. The bandwidth obtained with the solid immersion lens is almost double that in a configuration without this feature, and allowed us to obtain a good signal over a large spectral bandwidth (approximately $380 \mathrm{~nm}$ ). Moreover, the IOW spectrometer as developed here provides for easy and straightforward optical alignment of the coupling optics. As our protein experiments illustrate, the IOW spectrometer is extremely sensitive and reliable for probing ultrathin layers on the surface of a sensor platform. It is an excellent analytical tool with broadband spectroscopic capability that can be used for studying molecular adsorbates to address properties such as adsorption kinetics, surface coverage, molecular orientation, and molecular conformational changes, and to investigate those properties with respect to a variety of surface environments. The developments reported here are expected to have an important impact on the applications of single-mode integrated optical waveguide platforms for spectroscopic studies of surface-adsorbed ultrathin films and surface phenomena, as illustrated in one of our recent published works. $^{24}$

\section{Acknowledgments}

This project was funded by the NIH (award RR022864 to S.B.M.) and the NSF (award DBI-0352449 to S.B.M.).

\section{References}

1. I. Chabay, "Optical Waveguides: photon plumbing in the chemistry labs fiber optics, waveguides, and evanescent waves as tools for chemical analysis," Anal. Chem. 54(9), 1071A-1080A (1982).

2. P. W. Bohn, "Optical wave-guide techniques for structural and chemical analysis in thin-film and interfacial systems," TrAC Trends Anal. Chem. 6. 223-233 (1987).

3. K. Itoh and A. Fujishima, "An application of optical waveguides to electrochemistry: construction of optical waveguide electrodes," J. Phys. Chem. 92, 7043-7045 (1988).
4. S. S. Saavedra and W. M. Reichert, "Integrated optical attenuated total reflection spectrometry of aqueous superstrates using prism-coupled polymer wave-guides," Anal. Chem. 62, 2251-2256 (1990).

5. M. D. Degrandpre, L. W. Burgess, P. L. White, and D. S. Goldman, "Thin-film planar wave-guide sensor for liquid-phase absorbency measurements," Anal. Chem. 62, 2012-2017 (1990).

6. D. S. Goldman, P. L. White, and N. C. Anheier, "Miniaturized spectrometer employing planar wave-guides and grating couplers for chemical analysis," Appl. Opt. 29, 4583-4589 (1990).

7. S. S. Saavedra and W. M. Reichert, "In situ quantitation of protein adsorption density by integrated optical wave-guide attenuated total reflection spectrometry," Langmuir 7, 995-999 (1991).

8. K. Itoh and A. Fujishima, "An application of optical waveguides to electrochemical and photoelectrochemical processes," in Electrochemistry in Transition, O. J. Murphy, S. Srinivasan, and B. E. Conway, Eds., pp. 219-225, Plenum, New York (1992).

9. C. Piraud, E. K. Mwarania, J. Yao, K. Odwyer, D. J. Schiffrin, and J. S. Wilkinson, "Optoelectrochemical transduction on planar optical wave-guides," J. Lightwave Technol. 10, 693-699 (1992).

10. K. Kato, A. Takatsu, N. Matsuda, R. Azumi, and M. Matsumoto, "A slab-optical-wave-guide absorption-spectroscopy of LangmuirBlodgett films with a white-light excitation source," Chem. Lett., 437438 (1995).

11. S. B. Mendes, L. F. Li, J. J. Burke, J. E. Lee, D. R. Dunphy, and S. S. Saavedra, "Broad-band attenuated total reflection spectroscopy of a hydrated protein film on a single mode planar waveguide," Langmuir 12, 3374-3376 (1996)

12. D. R. Dunphy, S. B. Mendes, S. S. Saavedra, and N. R. Armstrong, "The electroactive integrated optical waveguide: ultrasensitive spectroelectrochemistry of submonolayer adsorbates," Anal. Chem. 69, 30863094 (1997)

13. J. T. Bradshaw, S. B. Mendes, and S. S. Saavedra, "A simplified broadband coupling approach applied to chemically robust sol-gel, planar integrated optical waveguides," Anal. Chem. 74, 1751-1759 (2002).

14. J. T. Bradshaw, S. B. Mendes, N. R. Armstrong, and S. S. Saavedra, "Broadband coupling into a single-mode, electroactive integrated optical waveguide for spectroelectrochemical analysis of surface-confined redox couples," Anal. Chem. 75, 1080-1088 (2003).

15. S. B. Mendes, J. T. Bradshaw, and S. S. Saavedra, "Technique for determining the angular orientation of molecules bound to the surface of an arbitrary planar optical waveguide," Appl. Opt. 43, 70-78 (2004).

16. J. T. Bradshaw, S. B. Mendes, and S. S. Saavedra, "Planar integrated optical waveguide spectroscopy," Anal. Chem. 77, 28A-36A (2005).

17. I. E. Araci, S. B. Mendes, N. Yurt, S. Honkanen, and N. Peyghambarian, "Highly sensitive spectroscopic detection of heme-protein submonolayer films by channel integrated optical waveguide," Opt. Express 15, 5595-5603 (2007).

18. S. B. Mendes, L. F. Li, J. Burke, and S. S. Saavedra, "Achromatic prismcoupler for planar waveguide," Opt. Commun. 136, 320-326 (1997).

19. S. B. Mendes, L. F. Li, J. J. Burke, J. E. Lee, and S. S. Saavedra, "70-nmbandwidth achromatic wave-guide coupler," Appl. Opt. 34, 6180-6186 (1995).

20. M. L. Dakss, L. Kuhn, P. F. Heidrich, and B. A. Scott, "Grating coupler for efficient excitation of optical guided waves in thin films," Appl. Phys. Lett. 16, 523-525 (1970).

21. E. Hecht, Optics, Addison Wesley (2001)

22. C. M. Hayes, M. B. Pereira, B. C. Brangers, M. M. Aslan, R. S Wiederkehr, S. B. Mendes, and J. H. Lake, "Sub-micron integrated grating couplers for single-mode planar optical waveguides," in UGIM 2008, pp. 227-232, IEEE (2008).

23. S. B. Mendes and S. S. Saavedra, "Comparative analysis of absorbance calculations for integrated optical waveguide configurations by use of the ray optics model and the electromagnetic wave theory," Appl. Opt. 39, 612-621 (2000)

24. R. S. Wiederkehr, G. C. Hoops, M. M. Aslan, C. L. Byard, and S B. Mendes, "Investigations on the Q and CT Bands of cytochrome c submonolayer adsorbed on an alumina surface using broadband spectroscopy with single-mode integrated optical waveguides," J. Phys. Chem. C 113, 8306-8312 (2009).

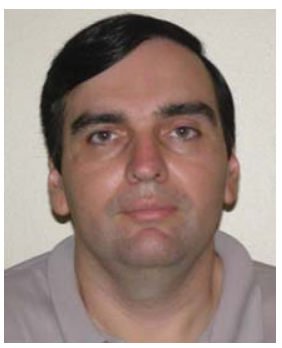

Marcelo B. Pereira received his $\mathrm{PhD}$ in material sciences from Universidade Federal do Rio Grande do Sul (UFRGS), Brazil, in 2003, and was a postdoctoral fellow at the University of Louisville, Kentucky, USA in 2006-2007. Presently, he is a professor at UFRGS, working on optical characterization of materials, ion-exchange waveguides, spectroscopy, and optical films. 
Pereira, Craven, and Mendes: Solid immersion lens at the aplanatic condition for enhancing the spectral bandwidth...

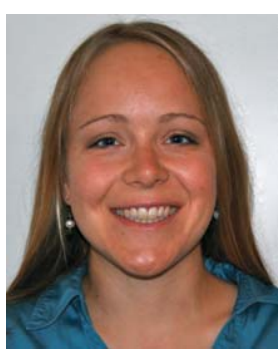

Jill S. Craven received her BS in chemical engineering at the University of Arizona in 2007. She is now working on a PhD in chemical engineering, specializing in the field of atmospheric aerosols, at the California Institute of Technology.

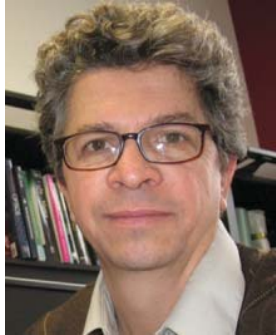

Sergio B. Mendes received his bachelor's degree in physics from the University of São Paulo, Brazil, in 1982. He then became a technical manager of Funbec, a private foundation inside the University of São Paulo focused on developing novel optical technologies in Brazil. In 1991, he went to the University of Arizona to pursue his $\mathrm{PhD}$ and got his degree in 1997, working under the supervision of Prof. James Burke. On his graduation, he became a faculty member of the College of Optical Sciences at the University of Arizona, a position that he held until joining the University of Louisville in August 2006 as an associate professor of physics. From 2000 till 2003, he had a leave of absence from his academic appointment to be a director of NP Photonics Inc., a spinoff company created to develop novel optical fiber amplifiers and fiber lasers. His current research work at the University of Louisville focuses on the development and applications of integrated optic, guided-wave, and plasmonic technologies for research in biomolecular films and surface phenomena, using several optical spectroscopic techniques. Prof. Mendes has been awarded several research grants by agencies such as the NSF, the $\mathrm{NIH}$, and NASA, has published more than 40 research articles in peer-reviewed journals, is the coinventor of five licensed patents, has been invited for several talks at international conferences, has supervised and collaborated in the research projects of several graduate and undergraduate students, and has served as a topical editor for the journal Applied Optics of the Optical Society of America. 